and swollen joints count $(p=0.001$ and $p=0.024$, respectively). Age and tender joints count were confirmed at multivariate analysis (OR 1.014, CI95\% 1.0011.027 and OR 0.951, CI95\% 0.923-0.981, respectively) as independent predictors of early withdrawal of MTX.

Conclusions: More than $75 \%$ of RA patients treated with MTX as first-line therapy remained in treatment after 12 months. Age was directly correlated and tender joints count was inversely correlated with early withdrawal of MTX in RA patients. Disclosure of Interest: None declared

DOI: 10.1136/annrheumdis-2018-eular.3812

\section{SAT0224 AMELIORATION OF INFLAMMATORY DISEASE ACTIVITY AND VASCULAR INFLAMMATION WITH HMG- COA REDUCTASE INHIBITION AND ANGIOTENSIN RECEPTOR BLOCKADE IN RHEUMATOID ARTHRITIS}

A. Syngle ${ }^{1,2}$, N. Garg ${ }^{3}$, P. Krishan ${ }^{3} .{ }^{1}$ Cardio Rheuma, Healing Touch City Clinic, chandigarh, ${ }^{2}$ Internal Medicine \& Rheumatology, Fortis Hospital, Mohali, ${ }^{3} 2$ Department of Pharmaceutical Sciences \& Drug Research, Punjabi Univ., Patiala, India

Background: Rheumatoid Arthritis (RA) has $50 \%$ increased risk of cardiovascu$\operatorname{lar}(\mathrm{CV})$ mortality ${ }^{1}$. Similarities between atherosclerosis and RA and proven benefit of Angiotensin receptor Blockers and HMG-CoA reductase inhibitors in atherosclerotic vascular disease provide strong rationale to investigate the impact of Rosuvastatin, HMG-CoA reductase inhibitor and Olmesartan, an angiotensin receptor blocker on inflammatory disease activity and vascular inflammation in RA.

Objectives: To investigate the impact of Rosuvastatin and Olmesartan on inflammatory disease activity and vascular inflammation in RA

Methods: 84 RA patients randomized to 3 groups to receive 24 weeks of treatment with Rosuvastatin (Rvs) (10 mg/day, $n=28)$, Olmesartan (OLME) (10 mg/ day, $n=28)$ and placebo $(P L)(n=28)$ as an adjunct to existing stable antirheumatic drugs. 2 patients from the OLME group were lost to follow up. FMD was assessed by AngioDefender. EPCs were estimated by flow cytometry. Measures of vascular inflammation: serum nitrite, TBARS, adhesion molecules (ICAM-1 and VCAM-1) and lipids were measured at baseline and after treatment. Inflammatory measures included DAS28, SDAI, CRP and ESR, pro-inflammatory cytokines (TNF- $\alpha$, IL-6 and IL-1). SCORE system estimated the 10 year risk of a first fatal atherosclerotic event. Quality of life was assessed with HAQ-DI and SF-36.
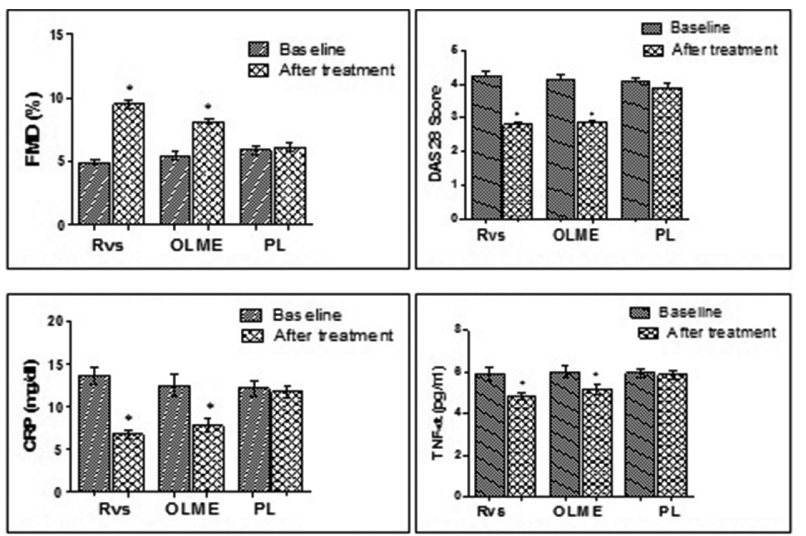

Figure 1A Effect on Flow Mediated Dilation (FMD).Rvs vs. OLME vs. PL (Rvs vs. PL $(p<0.01)$, OLME vs. PL ( $p \leq 0.01)$, Rvs vs. OLME ( $p=0.03)$ after 24 weeks. Figure $1 B$ Effect on Disease Activity Score of 28 joints (DA S28). Rvs vs. OLME vs. PL (Rvs vs. PL ( $p<0.01)$, OLME vs. PL. $(p \leq 0.01)$, Rvs vs. OLME $(p=0.08)$ after 24 weeks. Figure 1C Effect on C-Reactive Protein (CRP).Rvs vs. OLME vs. PL (Rvs vs. PL ( $p<0.01)$, OLME vs. PL $(p \leq 0.01)$, Rvs vs. OLME $(p=0.05)$ after 24 weeks. Figure 1D Effect on Tumour Necro sis Factor-alpha (TNF- $\alpha$ ). Rvs vs. OLME vs. PL (Rvs vs. PL $(p<0.01)$, OLME vs. PL $(p \leq 0.01)$, Rvs vs. OLME $(p=0.86)$ after 24 weeks.

Results: At baseline, FMD correlated inversely with DAS28 $(r=-0.42, p<0.05)$ and TNF- $\alpha(r=-0.50, p<0.05)$ and positively correlated with EPCs $(r=0.44$, $\mathrm{p}<0.05)$ in all three groups indicating high inflammatory disease activity and decreased EPCs population associated with endothelial dysfunction. FMD also correlated inversely with CRP in both Rvs $(r=-0.46, p<0.05)$ and OLME $(r=-0.40$, $\mathrm{p}<0.05)$ groups. After treatment, FMD improved significantly in the Rvs vs. OLME vs. PL group from their baseline levels, respectively \{Rvs vs. PL $(p<0.01)$, OLME vs. $P L(p \leq 0.01)$, Rvs vs. OLME $(p=0.03)\}$ (Fig.1A). The improvement in FMD after treatment with Rvs was significantly greater than OLME [Rvs vs. OLME $(p=0.03)$ ] EPCs and nitrite levels were improved significantly in both Rvs and OLME groups. A significant reduction was found in ICAM-1 after Rvs treatment $(p<0.01)$ where as OLME significantly decreased VCAM-1 and TBARs $(p=0.04),(p=0.01)$ respectively. Both Rvs and OLME resulted in significant reductions of DAS28 (figure 1B) SDAI, ESR, CRP (figure 1C), IL-6 and TNF- $\alpha$ (figure 1D) vs. PL. There was a significant reduction in the SCORE, HAQ-DI and SF-36 score after treatment with Rvs and OLME.

Conclusions: Rvs and OLME ameliorate inflammatory disease activity and vascular inflammation in RA. Both Rvs and OLME lowers the TNF- $\alpha$ \& IL- 6 which down regulates the production of $\mathrm{CRP}$ and $\mathrm{NO}$ and improved EPC population and FMD. However, Rvs also favourably impacted ICAM-1 and lipid abnormalities while OLME has beneficial effect on VCAM-1, TBARs and blood pressure. Thus, both Rvs and OLME ameliorate inflammatory disease activity, reduce cardiovas cular risk in context of vascular inflammation, endothelial dysfunction and EPCs biology.

\section{REFERENCE:}

1 López-Mejías R, Castañeda C, González-Juanatey C, et al. Autoimmunity Reviews 2016;15:1013-1030.

Disclosure of Interest: None declared DOI: 10.1136/annrheumdis-2018-eular.5605

\section{SAT0225 COMPARATIVE EFFECTIVENESS IN PAIN AND HAQ-DI IMPROVEMENT FOR BARICITINIB VERSUS ADALIMUMAB, TOCILIZUMAB, AND TOFACITINIB MONOTHERAPIES IN CSDMARD-NAÏVE RHEUMATOID ARTHRITIS PATIENTS: A MATCHING-ADJUSTED INDIRECT COMPARISON (MAIC)}

B. Fautrel ${ }^{1}$, B. Zhu ${ }^{2}$, P. C. Taylor ${ }^{3}$, M. van de Laar ${ }^{4}$, P. Emery ${ }^{5}$, F. De Leonardis ${ }^{2}$ C. Gaich ${ }^{2}$, C. Nicolay ${ }^{2}$, Z. Kadziola ${ }^{2}$, I. De La Torre ${ }^{2}$, R. Fleischmann ${ }^{6} .{ }^{1}$ University Pierre et Marie Curie, Paris, France, ${ }^{2}$ Eli Lilly and Company, Indianapolis, United States, ${ }^{3}$ Botnar Research Centre, Univ of Oxford, Headington, United Kingdom, ${ }^{4}$ Arthritis Center Twente, Enschede, Netherlands, ${ }^{5}$ Leeds MSK Biomed/Chapel Allerton Hosp, Leeds, United Kingdom, ${ }^{6}$ Univ of Texas Southwester Med Ctr, Dallas, United States

Background: In Phase 3 trial, RA-BEGIN, baricitinib (BARI) monotherapy demonstrated superiority to MTX in pain reduction and HAQ-DI improvement in treatment of cSDMARD-naïve active RA patients. ${ }^{1}$ No prospective head-to-head $(\mathrm{H} 2 \mathrm{H})$ trial data are available comparing BARI monotherapy vs. bDMARD monotherapy in csDMARD-naïve RA patients.

Objectives: To assess pain and HAQ-DI for BARI monotherapy from a randomized, MTX-controlled trial vs adalimumab (ADA), tocilizumab (TCZ), and tofacitinib (TOFA) monotherapy from similar randomized, MTX-controlled trials in csDMARD/bDMARD naïve RA patients using matching-adjusted indirect comparison (MAIC).

Methods: Individual patient data from the RA-BEGIN BARI $4 \mathrm{mg}$ arm were weighted to match baseline characteristics of the ADA arm from PREMIER, ${ }^{2}$ TOFA $5 \mathrm{mg}$ arm from ORAL-START ${ }^{3}$ and TCZ $8 \mathrm{mg} / \mathrm{kg}$ arm from combination of AMBITION and FUNCTION, ${ }^{4,5}$ respectively; MTX arms were also matched between trials. Method of moments was used to determine weights for age, gender, baseline disease scores, and baseline values of the outcome variable. Mean change on pain VAS and HAQ-DI at Week 24 for BARI were adjusted for the above baseline characteristics with the weighted linear model, and then indirectly compared vs. respective published results for Week $24 \mathrm{TCZ}$ and TOFA and for Week 26 ADA data. Statistical significance of the weighted treatment effect was assessed with the bootstrap method. Sensitivity analyses included MAIC with study level matching ${ }^{6}$, Bucher's method without matching adjustment ${ }^{7}$, and inclusion of disease duration as an additional matching variable.

Results: Across trials, the mean baseline pain VAS ranged from 58.7 to 65.2 with a 6-month mean change in pain of -28.3 to -33.5 for the MTX arm, indicating comparability between trials. Similar HAQ-DI and changes in $\mathrm{HAQ}$-DI for the MTX arm were observed. At Week 24, BARI showed numerically greater improvement over MTX in pain than that for TCZ, ADA, and TOFA; statistically significant pain improvement were observed for BARI vs ADA and TCZ with all 3 matching methods but only with the Bucher method for TOFA (figure 1). BARI-treated patients showed significantly greater improvement in HAQ-DI at Week 24 than TCZ and ADA but not TOFA (figure 1). Sensitivity analyses showed consistent results. 